\title{
Brief Understanding Alzheimer's Disease
}

\author{
Nahla Shaban khalil* \\ Critical Care Nursing Department, Faculty of Nursing Cairo University
}

*Corresponding Author: Nahla Shaban khalil, Critical Care Nursing Department, Faculty of Nursing Cairo University Email: Nahlakhalil28@yahoo.com

\section{EDITORIAL}

Alzheimer's disease is the most common form of dementia. There is no cure for Alzheimer's disease, nor any confirmed ways to prevent its occurrence. In Alzheimer's disease, brain cells start to deteriorate. The body tries to stop this process by forming a protein called amyloid. However, amyloid deposits expand in the brain, leading to further deterioration. These deposits of amyloid are named as "plaques" and make the brain cells to shrink leading to changes in the brain structure and cause the cells of the brain to die. The formation of plaques and knots also interfere with the formation of some essential brain chemicals, such as acetylcholine, which is important in memory function. Over time the loss of brain cells causes the brain to shrink.

Previous studies there are many factors may predispose to Alzheimer's disease; environmental factors such prolonged exposure to some environmental chemicals such as pesticides, glues and paints or exposure to viral or bacterial infection. As well, lifestyle factors, such as poor physical exercises, insomnia and lack of vegetables and fruits in diet may predispose to Alzehimer disease. Now, researchers believe that a combination of lifestyle, environmental and genetic risk factors may induce an abnormal biological process in the brain and results in Alzheimer. In addition, there are known risk factors for developing Alzheimer including old age, head injury, smoking, obesity, hypertension, uncontrolled diabetes.

The degenerative changes that happen with Alzheimer's disease alter the areas of the brain that master thought, memory and language causing gradual signs and symptoms that have connection with a person's behavior and mental function. As well, physical functions such as bladder and bowel control are also altered.
There is great individual variability regarding the attributes of symptoms experienced and the progress at which decline occurs. Behavior change and the length of symptoms are varied from person to other. The symptoms of Alzheimer's develop very slowly. The time between the onset of the disease and death can vary from 5 to 20 years.

The early symptoms that commonly occurred in Alzheimer's disease include: short-term memory loss, mood changes including irritability and anxiety, difficult learning new things, Loss of spontaneity, disorientation to time and place, communication difficulties, decline in ability to practice routine tasks. As Alzheimer's disease advances the following symptoms may develop: Increasing short-term memory loss, difficulty in recognizing family members and familiar friends, short attention span , restlessness, difficulty with reading, writing and numbers, disinterest and lack of hygiene, loss of appetite, personality changes eg: aggression, significant mood fluctuation , and needs assistance with daily tasks. Regarding the terminal stages of the disease the following symptoms may be experienced: Inability to comprehend or use speech, incontinence of urine / faeces, inability to recognize self or family, severe disorientation to time, increasing immobility and sleep time.

Diagnosis of Alzheimer disease involves complete assessment of medical and psychiatric history to exclude other possible causes. Therefore, a variety of tests are requested to attain accurate diagnosis, which may include: A neurological and physical examination, brain scans to evaluate brain structure and function that include CT scanning (computerized tomography), MRI (magnetic resonance imaging) and PET (positron emission tomography) and assessment of level of dependency. 
As there is no known cure for Alzheimer's disease, treatment focuses on managing symptoms of disease and counseling the person and their family. This may include: treating the possible causes that may progress to physical decline eg: lung disease or anemia ; encouraging arousing activities to reinforce the person to continue their habitual activities ; providing memory aids such as calendars and written reminders; encouraging social interaction to prevent feelings of loneliness and depression; and encourage regular routine to decrease confusion

Medications such as sleep helpers and tranquillizers may alleviate symptoms such as insomnia and restlessness. However, they often cause increased confusion, so their use should be restricted. Cholinesterase inhibitors drugs have shown some effectiveness in slowing the progression of the Alzheimer symptoms in some people. These medications assist in preventing the breakdown of acetylcholine, a neuro transmitter responsible for memory. Cholinesterase inhibitor medications that include donepezil, rivastigmine and galantamine. More recently, another drug memantine that works in a novel way to the cholinesterase inhibitors, aiming to prevent the entry of an increased amount of calcium into brain cells. Higher calcium levels in the brain cells causes damage and also prevents them from receiving signals from other brain cells. Other medications are researched and investigated for the treatment of Alzheimer's disease and include those that stop $t$ the formation of amyloid deposits in the brain, as well as use of some anti-inflammatory and hormone medications. As well, researches also investigate the use of alternative treatments such as anti-oxidants like Vitamin E, curcumin, selenium and some herbal extracts (ginko balboas in particular).
There are no proven ways to prevent the development of Alzheimer's disease. However, there is evidence to suggest that 1 a healthy lifestyle can reduce the risk of Alzheimer's disease. Regular physical activity and exercise may have a beneficial effect on brain health and may slow advancement of Alzheimer's disease. In addition, plant foods such as vegetables, fruits, beans, whole grains, nuts, olives and olive oil, yoghurt, and fish may decrease the risk of Alzheimer's disease, and decrease the incidence of cardiovascular disease and diabetes.

\section{REFERENCES}

[1] Alzheimer Society of Canada (2016). Stages of Alzheimer's Disease (Web Page). Toronto: Alzheimer Society Canada. www.alzheimer. ca/en/About-dementia/Alzheimer-s-disease/

Stages-of-Alzheimer-s-disease [Accessed:11/ 07/17]

[2] Alzheimer Society of Canada (2016). Alzheimer's disease (Web Page). Toronto: Alzheimer Society Canada. http://www. alzheimer.ca/en/About-dementia/Alzheimer-sdisease [Accessed: 11/07/17]

[3] Lakhan, S.E. (2017). Alzheimer disease (Web page). Medscape Drugs and Diseases. New York, NY: WebMD LLC.

[4] Mayo Clinic (2015). Alzheimer's disease (Web Page). Rochester, NY: Mayo Foundation for Medical Education and Research.

[5] Tobias, M, Yeh, L.C., Johnson, E. (2008). Burden of Alzheimer's disease: populationbased estimates and projections for New Zealand, 2006-2031. Aust NZ J Psychiatry; 42(9):828-36.

Citation: Nahla Shaban khalil. Brief Understanding Alzheimer's Disease, ARC Journal of Nursing and Healthcare. 2018; 4(1): 1-2. doi: dx.doi.org/ 10.20431/2455-4324.0401001.

Copyright: (c) 2018 Authors. This is an open-access article distributed under the terms of the Creative Commons Attribution License, which permits unrestricted use, distribution, and reproduction in any medium, provided the original author and source are credited. 